\title{
Cuidado Psicossocial em Saúde Mental em Contextos Rurais
}

\author{
Mauricio Cirilo da Costa Neto ${ }^{1}$ \\ Magda Dimenstein \\ Universidade Federal do Rio Grande do Norte, Natal, RN, Brasil
}

\section{Resumo}

As populações rurais sofrem historicamente efeitos do modelo socioeconômico pautado no latifúndio e exploração do trabalhador. Esse modelo está associado à produção de situações de vulnerabilidade psicossocial e ambiental que impactam negativamente na saúde mental. No meio rural há, portanto, certos condicionantes que são decisivos no processo saúde-doença e que devem ser considerados na produção da atenção. Este estudo teórico problematiza o cuidado em saúde mental em contextos rurais considerando o processo de determinação social da saúde e da vida, bem como os territórios concretos de existência das populações rurais. Realizou-se uma revisão da literatura nos principais bancos de dados, o que evidenciou a carência de estudos sobre a temática. Considera-se que a lógica territorial e comunitária que sustenta a Atenção Psicossocial precisa ser construída no cotidiano das equipes, visando respostas mais efetivas às necessidades de saúde mental das populações rurais, relacionadas diretamente às iniquidades que marcam a vida no campo.

Palavras-chave: Assistência integral à saúde, saúde da população rural, saúde mental, serviços de saúde mental, serviços de saúde rural.

\section{Psychosocial and Mental Health Care in Rural Environments}

\begin{abstract}
Rural populations have historically suffered the effects of a socio-economic model based on country estates and exploitation of workers. Such model is associated with the appearance of psychosocial and environmental vulnerabilities that have a negative impact on mental health. Therefore, in rural environments there are certain conditions that play a major role in the health-disease process and must be considered in health care planning. This theoretical study discusses the case of mental health care from the perspective of social determinants of health considering the concrete territories of rural populations. We conducted a literature review using relevant databases, which demonstrated a lack of studies about the chosen topic. The territorial and community logic supporting psychosocial care must be introduced in daily care services. Such process will provide more effective solutions to the mental health needs of rural populations, which are directly related to the iniquities that have shaped life in the countryside.
\end{abstract}

Keywords: Comprehensive health care, mental health, mental health services, rural health, rural health services.

Endereço para correspondência: Universidade Federal do Rio Grande do Norte, Rua Ary Parreiras, 224, Alecrim, Natal, RN, Brasil 59040-220. E-mail: mauricioneto_2@msn.com e mgdimenstein@gmail.com Financiamento: Coordenação de Aperfeiçoamento de Pessoal de Nível Superior (Capes). 


\section{Cuidado Psicosocial en la Salud Mental en Entornos Rurales}

\section{Resumen}

Las poblaciones rurales históricamente sufren efectos del modelo socio-económico basado en el latifundio y la explotación de los trabajadores. Este modelo se asocia con la producción de vulnerabilidades psicosociales y ambientales que impactan negativamente en la salud mental. En las zonas rurales, por lo tanto, existen ciertas condiciones que son decisivas en el proceso salud-enfermedad y deben ser consideradas en la producción de la atención. Este estudio teórico se ocupa de la atención en salud mental, teniendo en cuenta los determinantes sociales de la salud y la vida, así como las condiciones concretas de existencia de las poblaciones rurales. Se realizó una revisión bibliográfica en las bases de datos, lo que demuestra la falta de estudios sobre el tema. Se considera que la lógica territorial y comunitaria que orienta la atención psicosocial debe hacer parte de la vida diaria de los equipos, buscando respuestas más eficaces a las necesidades de salud mental de las poblaciones rurales, directamente relacionadas con las desigualdades sociales que marcan la vida en el campo.

Palabras clave: Atención integral de salud, salud mental, salud rural, servicios de salud mental, servicios de salud rural.

O cuidado em saúde mental é uma temática complexa e multideterminada que envolve uma diversidade de aportes teórico-metodológicos e implica em posicionamentos ético-políticos. Refletir sobre o cuidado em saúde mental atentando para as condições de vida e saúde das populações rurais, para os modos de sociabilidade e convivência que marcam as relações cotidianas nesses cenários, bem como para os saberes e práticas tradicionais e recursos informais que fazem parte do arsenal terapêutico dessas populações, levanta uma série de desafios teóricos-metodológicos para a atenção e gestão em saúde mental no Sistema único de Saúde (SUS).

Esses desafios dizem respeito ao fato de que as populações rurais não são homogêneas, há diferentes dinâmicas e sujeitos sociais, mobilidades populacionais, relações sociais, modos de produção, aspectos culturais e ambientais, povos e comunidades que têm seus modos de vida e reprodução social relacionados com o campo, com a floresta e com os ambientes aquáticos. Ou seja, são camponesas(es); agricultoras(es) familiares; trabalhadoras(es) rurais assentadas(os) ou acampadas(os); trabalhadoras(es) assalariadas(os) e temporárias(os); comunidades tradicionais, como quilombolas, ribeirinhas(os); pescadoras(es) ar- tesanais e marisqueiras; as que habitam e utilizam reservas extrativistas em áreas florestais ou aquáticas; aquelas atingidas por barragens; dentre outras (Silva et al., 2015).

O modelo socioeconômico hegemônico produz custos sociais que impactam na vida, trabalho e na saúde dessas populações. As condições de trabalho no campo vêm conformando um quadro epidemiológico preocupante caracterizado pela alta prevalência de morbidades do sistema osteomuscular, transtornos mentais, bem como outras relacionadas ao uso crescente de insumos químicos e inadequação de instrumentos de proteção que causam acidentes e graves processos de intoxicação. As precárias condições de vida das populações do campo e da floresta estão relacionadas aos agravos em saúde como parasitoses intestinais e diarreias, decorrentes da carência de saneamento básico e acesso à rede hídrica; taxas elevadas de mortalidade infantil; além de doenças infectocontagiosas como a doença de Chagas e a leishmaniose (Ministério da Saúde, 2011).

O debate sobre atenção em saúde ainda é urbano e etnocêntrico, isto é, não é sensível às peculiaridades socioculturais de outros contextos. Isso é reflexo do colonialismo de saberes e 
práticas no campo da saúde, o que contribui para a manutenção das iniquidades que marcam a história de vida das populações rurais, por exemplo. Este trabalho ${ }^{2}$ discute o cuidado em saúde mental considerando o processo de determinação social da saúde e da vida, bem como os territórios concretos de existência das populações rurais. Considera-se que a lógica territorial e comunitária que orienta a Atenção Psicossocial precisa ser construída no cotidiano das equipes, visando respostas mais efetivas às necessidades de saúde mental das populações rurais, relacionadas diretamente às iniquidades sociais que marcam a vida no campo.

\section{Método}

Realizou-se uma revisão sistemática sobre a temática do cuidado em saúde mental em contextos rurais nas seguintes bases de dados: SciELO Brasil, Biblioteca Virtual em Saúde (BVS) Brasil, e a Biblioteca Digital Brasileira de Teses e Dissertações (BDTD). Foram escolhidas por serem consideradas bases de dados virtuais de referência para publicações nacionais. A SciELO é uma biblioteca eletrônica que abrange uma coleção selecionada de periódicos científicos brasileiros. A BVS Brasil é parte integrante da Biblioteca Virtual em Saúde para América Latina e Caribe. Já a BDTD é um portal que integra os sistemas de informação de teses e dissertações existentes nas instituições de ensino e pesquisa brasileiras. Esta pesquisa foi realizada nas três bases separadamente, sem critério de exclusão, já que se trata de temática pouco publicada. Os tipos de documentos encontrados foram artigos, livros e capítulos de livros. É importante salientar ainda que ao longo da articulação teórica destacou-se algumas questões do campo de pesquisa que confirmam elementos apontados pela literatura no assunto.

2 Este trabalho é fruto de pesquisa de Mestrado que teve como objetivo geral caracterizar e analisar o cuidado em saúde mental ofertado à moradores de assentamentos rurais do estado do Rio Grande do Norte.

\section{Vulnerabilidades Psicossocial e Ambiental e Saúde Mental no Campo}

A reprodução da pobreza rural está diretamente relacionada ao modelo socioeconômico que garante os privilégios das grandes empresas voltadas ao agronegócio, as quais organizam o espaço em torno de sua atividade produtiva, reproduzindo o latifúndio e a exploração do trabalhador rural como pilares fundamentais da vida econômica e social do campo. Neste sentido, a pobreza impacta sobremaneira as populações rurais, constituindo um cenário onde cerca de $50 \%$ da população rural vive nestas condições ou enfrenta situações precárias de vida, conforme estudos que reiteram a predominância da pobreza em zonas rurais (Kageyama \& Hoffmann, 2006; Rocha, 2006).

Apesar da persistência da pobreza no meio rural, nas duas últimas décadas ocorreu crescimento relativamente forte da renda, bem como queda acentuada da desigualdade nos contextos rurais (Helfand, Rocha, \& Vinhais, 2009). A incipiente melhora das condições de vida das populações do campo, resultado das políticas agrárias, serviu mais para atenuar as situações de conflito social do que para modificar as situações de pobreza e desigualdade social. Assim, os assentados carregam uma trajetória de vida marcada pela precária condição de reprodução social, grande dificuldade de acesso às políticas e programas de saúde, educação, segurança, transporte, habitação e organização da produção (Dimenstein et al., 2014). Diante disso, podemos inferir que o modo como os assentamentos rurais $^{3}$ estão configurados na atualidade estão pro-

3 O termo "assentamento" apareceu pela primeira vez no vocabulário jurídico e sociológico no contexto da reforma agrária venezuelana, em 1960, e se difundiu posteriormente para outros países. Utilizando o conceito de Bergamasco e Norder (1996, p. 07), nós entendemos os assentamentos rurais como "unidades de produção agrícola, criados por meio de políticas governamentais, visando o reordenamento do uso da terra, em benefício de trabalhadores rurais sem terra ou 
duzindo situações de vulnerabilidade psicossocial e ambiental ${ }^{4}$.

As particularidades sociais, políticas e territoriais do campo brasileiro estão associadas a uma série de fenômenos como a devastação de florestas, extinção da biodiversidade, erosão de terras, mas também com a precarização das relações de trabalho no campo, com os fluxos migratórios para as cidades, etc. Tudo isso constitui um cenário de vulnerabilidade psicossocial e ambiental que impacta de modo mais incisivo na vida e na saúde das populações com escassez de bens e recursos sociais e materiais para o enfrentamento desses fatores de risco (Pinheiro, Silva, Carneiro, Faria, \& Silva, 2009).

Nesta perspectiva, a pobreza, desigualdades no acesso à terra e fatores ambientais têm disseminado efeitos psicossociais nas populações do campo. A fome, violência, traumas, dor, humilhação, e falta de reconhecimento vividos por segmentos sociais subalternizados podem se configurar como fatores mediadores de sofrimentos variados. Logo, a privação múltipla de bens e serviços impacta negativamente na saúde mental da população (Silva \& Santana, 2012).

Estudos sobre saúde mental em populações rurais são escassos em nível internacional (Ministerio de Sanidad y Política Social, 2011). No Brasil, um dos poucos estudos nesse sentido, o relatório da Confederação Nacional dos Trabalhadores da Agricultura (CONTAG, 2013), de-

com pouca terra" e são fruto tanto de políticas agrárias federais, como estaduais e municipais.

4 Para nos auxiliar a compreender a situação de vulnerabilidade social, enquanto formas diversas de exclusão ou alijamento de grupos populacionais àqueles fatos ou benefícios que possam estar acontecendo no processo desenvolvimentista mundial, podemos ampliar a noção de vulnerabilidade no sentido de concebê-la enquanto vulnerabilidade socioambiental. Essa concepção trata da coexistência ou sobreposição espacial entre coletivos em situação de pobreza e com alta privação que convivem em áreas de risco ou degradação ambiental (Cartier, Barcellos, Hübner, \& Porto, 2009), Desse modo, os grupos sociais vulneráveis ao conviverem com fatores de risco existentes no território produzem vulnerabilidades socioambientais. tectou como principais agravos os problemas de coluna, hipertensão, dores de cabeça constantes, disfunções gastrointestinais, alergia/problema de pele e insônia, ou seja, queixas que apontam para a possibilidade de sofrimento de base psicossocial que estão associadas às condições de vida e trabalho no campo.

A Organização Mundial de Saúde (World Health Organization [WHO], 2010) considera que as situações de vulnerabilidade produzem impactos psicossociais na população. $\mathrm{O}$ estigma e a marginalização podem impactar a autoestima, autoconfiança, reduzir a motivação e interferir nos projetos de vida. $\mathrm{O}$ isolamento e a exposição cotidiana à violências e abusos podem causar sintomas psicossomáticos, uso de substâncias psicoativas, bem como problemas de saúde mental graves. Da mesma forma, a saúde mental é impactada negativamente quando ocorre violação dos direitos civis, culturais, políticos e sociais, ou ainda quando os grupos sociais são excluídos das oportunidades de geração de renda ou educação, o que traz implicações particulares para as populações rurais que sofrem historicamente com situações de pobreza. Como agravante das situações de vulnerabilidade, os problemas de saúde mental vivenciados pelos grupos comumente não encontram respostas $\mathrm{e}$ soluções pelos sistemas nacionais de saúde.

Autores como Costa, Dimenstein e Leite (2014) em seu estudo com assentados consideram que a combinação de um conjunto de riscos socioeconômicos e ambientais impactam a qualidade de vida da população e incidem sobre as condições de saúde, produzindo sofrimentos como transtornos mentais comuns. Há um conjunto de problemas, estressores e características específicas no meio rural associados aos problemas de saúde mental, o que significa que a resolução dos agravos depende da articulação de uma variedade de fatores existentes nos territórios que produzem experiências de sofrimento diferenciadas. Compreender o cuidado em saúde mental, a partir das dinâmicas socioterritoriais, das condições de vida e trabalho implica em atentar para os processos de determinação social da saúde das populações rurais. 


\section{Tessituras do Cuidado Psicossocial: Determinação Social da Saúde e da Vida}

Atualmente no campo da saúde mental a Atenção Psicossocial é considerada a estratégia norteadora na organização dos serviços e no direcionamento das práticas de cuidado na comunidade e no território (Amarante, 2007). Contudo, é preciso problematizar em que medida os avanços implementados por esta nova lógica de funcionamento, que se pretende paradigmática, estão realmente respondendo as necessidades de saúde relacionadas aos territórios singulares de vida das populações.

Considerar o cuidado em uma perspectiva psicossocial implica no alargamento do conceito de saúde, contemplando a variedade de determinações que produzem os processos de saúde-doença. De acordo com Basaglia (1979), o sofrimento mental é também uma questão de desigualdade, opressão, intolerância, de marginalização e exclusão de sujeitos e populações. Nesses termos, ao ancorar o cuidado na ótica da determinação social da saúde e da vida, as práticas de cuidado não podem se restringir às variáveis biomédicas e/ou psicológicas.

A produção teórica sobre a Determinação Social da Saúde (DSS) pode fornecer elementos para o estabelecimento de cuidados conectados com o cotidiano de vida das populações. Historicamente, o debate sobre a determinação social introduziu no campo da saúde uma série de críticas ao tecnicismo, pragmatismo e reducionismo dos modelos de compreensão do processo saúde-doença, bem como ao conhecimento produzido de modo a-histórico e descontextualizado do cotidiano de vida das populações (Almeida-Filho, 2010; Bosi, Melo, Carvalho, Ximenes, \& Godoy, 2014; Buss \& Pellegrini, 2007; Fleury-Teixeira \& Bronzo, 2010; Garbois, Sodré, \& Dalbello-Araújo, 2014; Garrafa \& Cordon Portillo, 2009; Souza, Silva, \& Silva, 2013; Tambellini \& Schutz, 2009).

O conceito de determinantes passou a ser amplamente utilizado nas produções teóricas e por organismos nacionais e internacionais para pensar o processo saúde-doença-cuidado e ex- pressa, de modo generalizado, que as condições de vida e trabalho dos indivíduos e de grupos populacionais interferem em sua situação de saúde. Os determinantes são utilizados para compreender o processo de produção de iniquidades em saúde das populações, conforme destaca a OMS (WHO, 2003) e direcionar resoluções de políticas públicas: desigualdade social, estresse, os primeiros anos de vida, exclusão social, trabalho, desemprego, suporte social, dependência química, alimentação e transporte.

Apesar de destacar certas condições sociais que se configuram como determinantes da saúde, os estudos sobre os DSS se restringem a apontar correlações causais entre fatores sociais e os fenômenos da saúde. Fundamentado na epidemiologia tradicional que se propõem a identificar as relações entre variáveis sociais e eventos de morbimortalidade entre os diferentes grupos da população, essa categoria analítica fortalece a perspectiva positivista a qual inicialmente questionou (Almeida-Filho, 2010; Nogueira, 2010).

A categoria "determinante" em seu caráter causal, linear e mecânico, desconsidera outras modalidades de produção do processo saúde-doença-cuidado, como processos dialéticos, cíclicos, estruturais, dentre outros. Reconhecer os diversos fatores que impactam no processo saúde-doença das populações é insuficiente para pensar a complexidade da saúde, o que torna necessário apreender a determinação social da saúde como processo de construção histórico e cultural das condições de vida e suas múltiplas determinações (Almeida-Filho, 2010; Breilh, 2010; Fleury-Teixeira \& Bronzo, 2010; Garbois et al., 2014).

Compreende-se o processo saúde-doença-cuidado das populações e indivíduos em sua multideterminação, inserido em contexto sociocultural, influenciado por uma complexa interação entre elementos que variam desde níveis micro até níveis macro. Nessa perspectiva, a categoria território emerge como noção basilar para pensar o cuidado psicossocial. Tornar o território princípio ordenador das estratégias de cuidado significa estabelecer relação íntima dos serviços com o contexto social e de existência concreta dos sujeitos. Nesse sentido, estruturar 
serviços, programas e desenvolver ações de cuidado sob uma lógica territorial implica em "olhar e ouvir a vida que pulsa nesse lugar" (Lima \& Yasui, 2014, p. 597). Dito isso, inferimos que a determinação social da saúde é agenciada nos territórios, em meio as materialidades existentes nos contextos de vida, das sociabilidades constituídas, das existências que se fazem possível (Koga, 2015).

\section{Tessituras do Cuidado Psicossocial: Construindo uma Lógica Territorial de Cuidado}

As elaborações teóricas sobre o território têm como bojo a Geografia, com destaque para a obra de Milton Santos, para quem a análise do território se torna um conceito utilizável para a análise social, quando o consideramos a partir de seu uso e juntamente com os atores que dele se utilizam. A concepção de território vivo do autor compreende um lugar de construção de relações pessoais, sociais, políticas e culturais que influenciam os modos de vida das pessoas que o habitam. Como produto e produtor das relações sociais, os territórios são objetos e agentes de transformações sociais, ou seja, ganham sentido pelo habitar de seus moradores (Santos, 2008).

Utilizar a categoria território como subsídio para problematizar o cuidado psicossocial significa que tratamos de modos de cuidado que estejam conectados com os contextos sociais onde a vida transcorre, espaço onde os serviços devem estar ancorados e o chão sobre o qual serão produzidos os modos de cuidado.

Tornar o território um princípio organizador das práticas de cuidado exige a relação íntima com o contexto social dos usuários, de sua existência concreta, para assim, poder produzir saúde em meio a condições sociais estabelecidas que podem operar na produção de adoecimento. Nesse sentido, é inescusável ampliar a percepção e descentrar o olhar dos sistemas de cuidado fisiológicos e psicológicos em direção à complexidade movente do território, seus contextos peculiares e interações. Imprescindível, não obstante, cultivar modos de apreender as necessidades de saúde das populações e conside- rar os determinantes postos nas localidades por meio da mediação de saberes técnicos, populares e tradicionais (Almeida, 2015).

Quando a população assentada consegue vencer as dificuldades de acesso aos serviços de saúde e assistência social, são assistidas por modelos de atenção que, invariavelmente, não são sensíveis às suas particularidades. Para Rigotto et al. (2012), os serviços de saúde apresentam problemas no acolhimento e cuidado desenvolvido diante das necessidades de saúde das populações rurais, pois não consideram as especificidades dos processos produtivos, modos de vida, formas de sociabilidade e convivência existentes nos territórios no planejamento das ações, orientando suas práticas pelos programas verticais e descontextualizados dos ministérios responsáveis.

As discussões em torno do território devem nortear a implementação dos serviços de saúde nos contextos rurais. Essa organização deve ser guiada pelas materialidades, fluxos e usos dos territórios, visando a integralidade do cuidado. Dessa forma, uma cidade de pequeno porte do interior do semiárido nordestino difere em termos de organização de rede das localidades na região amazônica ou das periferias de uma metrópole (Guimarães, Pickenhayn, \& Lima, 2014). O modelo de organização da rede de serviços e ações interfere diretamente na acessibilidade de grupos populacionais distantes dos centros urbanos, como ocorre com as diversas populações rurais.

Seguindo essa linha de pensamento, experiências na Espanha tentam lidar com as especificidades socioterritoriais e podem servir de inspiração para o ordenamento do cuidado em saúde mental em contextos rurais. Nesse país, as soluções delineadas foram o desenvolvimento de programas flexíveis organizados em equipes pequenas e próximas do cotidiano de vida das pessoas, indo além dos tradicionais equipamentos de saúde mental marcados pela rigidez do processo de trabalho no que concerne à circulação de profissionais, horários de funcionamento do serviço, protocolos de encaminhamento tradicionais, e profissionais locados no serviço. Vale destacar que a organização das redes de cuidado em contextos rurais naquele país tem por prin- 
cípio o atendimento das necessidades de cada território, o que produz serviços, programas e estratégias de cuidado locais e singulares.

No entanto, alguns elementos organizam o processo de trabalho nesses serviços, como: (a) existência de protocolos para detecção dos usuários em contextos rurais, evitando os vazios assistenciais; (b) garantia de no mínimo 20\% da carga horária dos profissionais reservada para ações nos territórios; (c) programas de atenção domiciliar tendo em vista os contextos rurais; (d) alta coordenação entre os serviços de saúde para os casos mais isolados geograficamente, com destaque para a atenção primária; (e) potencialização da atenção primária na coordenação do cuidado, bem como a utilização dos equipamentos sociais existentes nos territórios; (f) intervenção com as famílias de áreas mais distantes como modo de obter colaboração nos cuidados do usuário; e (g) capacitação e formação continuada dos profissionais para intervir nestes contextos, contribuindo para qualificar os programas e intervenções psicossociais (Ministerio de Sanidad y Politica Social, 2011).

A categoria território nos serve sobretudo para pensar as práticas de cuidado no cotidiano de serviços nesses contextos. Quando compreendemos que as necessidades de saúde são produzidas de acordo com os territórios de existência concreta das populações, desnaturalizamos a compreensão sobre as condições de vida, os problemas de saúde e consideramos os fatores socioambientais que interferem nessas condições. Outros modos de cuidado podem ser agenciados, não mais restritos aos agravos à saúde e conjunto de sintomas dos indivíduos. Além disso, permite causar interferência no modelo tecnoassistencial biomédico que define uma assistência individualista e curativista, que incentiva a procura desenfreada do serviço para restabelecimento da saúde e cria um ciclo de oferta e demanda por esse tipo de assistência pontual (Franco \& Merhy, 2013).

Para Ayres (2014) é preciso superar as perspectivas reducionistas acerca do processo saúde-doença das populações em condição de vulnerabilidade. A complexidade dos processos implicados nas condições de saúde destas populações exige outros modos de compreensão que não são compreensíveis à luz do paradigma biomédico hegemônico da saúde. O autor aposta em arranjos tecnoassistenciais sensíveis às diversidades e peculiaridades das necessidades de saúde, e, portanto, potentes para responder adequadamente a elas.

Nesse sentido, Merhy (2013) aposta na coletivização da gestão dos processos de trabalho em saúde, tomando como referência o interesse dos usuários e suas necessidades singulares de saúde. Considera que as tecnologias leves, que se referem às tecnologias relacionais, construídas nos espaços intercessores criados entre trabalhadores e usuários, podem coordenar as práticas de saúde, o que institui uma clínica das singularidades e dos processos emancipadores. Nesse sentido, produzir espaços dialógicos entre os atores envolvidos, possibilita apreender as peculiaridades dos modos de vida no campo, a história de luta pela reprodução social e constituição de sujeitos políticos; os diversos modos de trabalho na terra; as sociabilidades que se estruturam; as redes sociais que compartilham; os modelos familiares que escapam da família tradicional burguesa; as crenças e religiosidades; as concepções diferenciadas de saúde e práticas populares de cuidado; retirando as populações de um território estático, identitário e homogeneizador, colocando-os em um território processual, dinâmico e passível de transformação.

\section{Cuidado Psicossocial em Saúde Mental em Contextos Rurais: Atenção Primária em Destaque}

Levando em consideração os índices negativos de saúde das populações do campo e a carência de políticas públicas voltadas para esse segmento, a XII Conferência Nacional de Saúde em 2004 apontou como um dos principais desafios do SUS a garantia do acesso ao sistema de saúde pela população rural (Bergamaschi, Teles, Souza, \& Nakatami, 2012). Nesse caminho o Ministério da Saúde instituiu o Grupo da Terra, que trabalhou na elaboração da Política Nacional de Saúde Integral das Populações do Campo das Florestas e das Águas (PNSIPCFA) publicada no ano de 2011, e que busca levar em conta as 
especificidades das diferentes populações rurais brasileira visando o acesso integral e universal à saúde, além de introduzir nos programas de saúde já existentes as necessidades específicas em saúde dessas populações.

Em relação à saúde mental, ao analisarmos a PNSIPCFA verificamos que não há nenhuma novidade que provoque alterações nos princípios e na Rede de Atenção Psicossocial (RAPS) no sentido de considerar as especificidades socioterritoriais e psicossociais dessas populações. A política busca fortalecer a RAPS e tece comentários sobre aspectos epidemiológicos associados ao trabalho no campo como disparadores de sofrimentos. Do mesmo modo, na política de saúde mental em vigor não há uma diretriz estabelecida de atenção e cuidado que consiga atender às especificidades das populações do campo. Diante das peculiaridades socioterritoriais das populações do campo, as necessidades de saúde destes povos exigem uma paradoxal radicalização dos próprios princípios que conformam a atenção psicossocial no país. Se o seu sentido passa por uma transformação de relações de poder, de novas articulações entre atores e da desconstrução dos saberes, práticas e culturas do modelo asilar, agora são os saberes, práticas e culturas próprios de seu modelo que são desafiados diante de singularidades outras associadas a estas populações (Assis et al., 2014).

Apesar da ausência de referência direta à saúde mental, dentre as principais diretrizes da PNSIPCFA, está a valorização das "práticas e conhecimentos tradicionais, com a promoção do reconhecimento da dimensão subjetiva, coletiva e social dessas práticas e a produção e reprodução de saberes das populações tradicionais" (Ministério da Saúde, 2011, p. 6). A valorização do saber popular e das práticas tradicionais de saúde expressa o compromisso com a produção e reprodução de saberes das populações tradicionais, dentre estes, os saberes de parteiras, benzedeiras, raizeiras, xamãs, e etc.

Mesmo alvo de processos de marginalização social, as populações rurais mantêm saberes e práticas que são produtores de pertencimento e que organizam os seus modos de compreender e agir na vida. $\mathrm{O}$ aspecto cultural, sejam as crenças populares, religiosidade, alimentação e sociabilidades, estrutura modos próprios de responder aos processos de adoecimento, servindo para amenizar as dores e sofrimentos (Budó \& Saupe, 2005; Escola de Saúde Pública de Minas Gerais, 2014; Lara, Brito, \& Rezende, 2012). A potencialização das práticas culturais e informais de cuidado torna-se um foco de ação importante para os profissionais que atuam nesses contextos, tendo em vista que estas são as primeiras iniciativas tomadas pelos usuários frente aos processos de adoecimento (Silva, Dimenstein, \& Leite, 2013).

Além da valorização dos saberes tradicionais, a comunidade representa para a atenção psicossocial um importante lócus de investimento das ações em saúde. Os trabalhadores podem agir no sentido de subsidiar as iniciativas comunitárias que servem de suporte e apoio aos sujeitos. Juliano e Yunes (2014) concebem as redes de apoio social como coletivos de sistemas e pessoas significativas que compõem os elos de relacionamentos recebidos e percebidos pelos sujeitos. A afetividade é peça crucial, em função do seu incontestável valor para a constituição e manutenção das redes de apoio. Diante das condições de pobreza e vulnerabilidade que vivenciam parte das populações rurais, a eficácia das redes de apoio pode funcionar como mecanismo de enfrentamento às condições adversas de vida, bem como de proteção social.

De acordo com Vasconcelos (2013), as cooperativas comunitárias e associações de trabalhadores rurais, os movimentos sociais do campo, até mesmo grupos de base religiosa e rodas de vizinhança podem se configurar como grupo de ajuda e suporte mútuo, pois reúne a troca de vivências, o compartilhamento de histórias de vida, ajuda emocional, aconselhamento e discussão de estratégias para enfrentar os problemas cotidianos (ajuda mútua), acrescidos a isso ações de cuidado e suporte concreto (suporte mútuo). Para Vasconcelos (2013) tais grupos e redes de amizade constituem importantes estratégias de empoderamento, ou seja, de fortalecimento, aumento de poder e autonomia pessoal e coletiva perante as situações de opressão, violência ou discriminação. $\mathrm{O}$ estabelecimento e manutenção 
dos laços sociais entre as pessoas que compartilham do mesmo espaço de uma comunidade indica maior coesão social, trocas recíprocas e suporte social, fatores de proteção contra os processos adoecedores (Almeida, 2015).

Nesta perspectiva, pensando nas populações rurais, a atenção primária em saúde se destaca como nível de atenção que permite imersão no território onde as populações vivem, o reconhecimento das necessidades de saúde e dos processos de determinação social da saúde. Por meio do estabelecimento de vínculos e responsabilização pelos usuários, abre possibilidades de cuidado mais relacionais e singularizadas, contribuindo para respostas mais resolutivas e condizentes com os problemas locais.

Alguns estudos em áreas rurais (Oliveira et al., 2015; Uchoa et al., 2011) sobre o acesso aos cuidados primários e qualidade da atenção às populações rurais destacam que houve melhora significativa das condições de vida da população, a partir da expansão da atenção primária, principalmente no que diz respeito ao acompanhamento do pré-natal, do crescimento e desenvolvimento infantil, ao controle de diabetes e hipertensão, e, finalmente, à expansão do atendimento odontológico. Quando se trata da saúde mental, os estudos são escassos, todavia, algumas experiências observadas na Espanha notadamente concluem sobre a relevância da atenção primária na atenção às populações que se encontram distantes dos centros urbanos, como é o caso das populações rurais (Ministerio de Sanidad y Politica Social, 2011).

Em seu estudo na atenção primária em contextos rurais, Oliveira et al., (2015) perceberam equipes de atenção primária pautando suas ações em perspectivas interdisciplinares e intersetoriais, com maior participação dos usuários na organização da atenção e nos planos de ação comunitários. Destacaram ainda que, ao compreenderem a relação direta entre saúde e a terra, enquanto condição material e simbólica de vida dessas populações, as equipes vêm construindo ações de fortalecimento de equipamentos e serviços coletivos de apoio às famílias, como moradia, sistemas de telefonia, mobilidade, que podem interferir de modo decisivo nas condições de vida e saúde, bem como na permanência das famílias no campo.

Nesse sentido, destacamos que o cuidado no âmbito primário possibilita articular uma rede comunitária de cuidados, conectando diferentes equipamentos sociais e institucionais. Uma rede desta natureza abre caminho não apenas para a modificação das formas de cuidado, acolhimento e suporte aos usuários, mas também permite a invenção de novas relações e sociabilidades nos espaços de vida comunitário. Dimenstein (2013), tendo como referência experiências internacionais, relata a potência dos cuidados primários no sentido da complexificação e ampliação das estratégias de cuidado, contemplando questões como a convivência comunitária e o trabalho. Em outras palavras, a inserção da saúde mental na APS se faz necessária para a sustentabilidade da própria Reforma Psiquiátrica (Dimenstein, Lima, \& Macedo, 2013; Figueiredo \& Campos, 2009).

A maior parte dos municípios do país não preenche os critérios populacionais (não possui 15.000 habitantes) que sustentem a implementação de dispositivos como o CAPS, o que significa que a atenção primária é a referência mais importante no atendimento em saúde mental para grande parte das populações rurais. Esse nível assistencial é para onde convergem diversas políticas públicas, como a Política Nacional de Saúde Integral dos Povos do Campo, das Florestas e das Águas (PNSIPCFA), de saúde mental, de assistência social etc. As ações da atenção primária permitem contemplar a saúde em sua integralidade, reconhecer os determinantes que interferem nas condições de saúde, identificar os nós mais sensíveis onde intervenções podem produzir maior impacto sobre a saúde das populações e atacar os diversos fatores que impactam e dificultam a vida das famílias e comunidades (Buss \& Pellegrini, 2007).

\section{Considerações Finais}

Esse estudo detectou pouca produção nacional e internacional em termos do cuidado em saúde mental voltado às populações do campo. A atenção em saúde e à saúde mental tem sido 
marcada pela tecnificação, objetificação, biologicismo, mecanicismo, hospitalocentrismo, curativismo e especialismo. A perspectiva da determinação social da saúde e da vida, bem como os estudos sobre o território, fornecem subsídios teóricos-metodológicos que podem contribuir para uma compreensão ampliada do processo saúde-doença-cuidado, especialmente em contextos rurais, potencializando a lógica da atenção psicossocial que orienta a reforma psiquiátrica brasileira. Dessa forma, o cuidado psicossocial em saúde mental possui algumas características: é produzido no território, é sustentado por tecnologias de cuidado singulares, contextualizadas, culturalmente sensíveis e eticamente comprometidas com a vida das populações rurais. Nesse sentido, tem forte potencial de contribuir no enfrentamento das iniquidades em saúde, das vulnerabilidades sociais e ambientais historicamente vivenciadas no campo. Por fim, destacamos a Atenção Primária como o nível de atenção onde o cuidado psicossocial em saúde mental apresenta mais possibilidades de ser coproduzido entre trabalhadores e moradores do campo, a partir da articulação de saberes e tecnologias.

\section{Referências}

Almeida, K. S. de. (2015). Urbanização e modos de vida: Debate sobre determinação social da saúde (Dissertação de mestrado, Universidade Federal do Rio Grande do Norte, Natal, RN, Brasil).

Almeida-Filho, N. (2010). A problemática teórica da determinação social da saúde. In N. Nogueira (Ed.), Determinação social da saúde e reforma sanitária (pp. 13-36). Rio de Janeiro, RJ: Centro de Estudos Brasileiros de Saúde.

Amarante, P. (2007). Saúde mental e atenção psicossocial. Rio de Janeiro, RJ: Fundação Oswaldo Cruz.

Assis, J. T. de, Barreiros, C. A., Jacinto, A. B. M., Kinoshita, R. T., de Lemos Macdowell, P., \& Mota, T. D. (2014). Política de saúde mental no novo contexto do Sistema Único de Saúde: Regiões e redes. Saúde em Debate, 52, 88-113.

Ayres, J. R. (2014). Vulnerabilidade, direitos humanos e cuidado: Aportes conceituais. In S. Bar- ros, P. F. S. Campos, \& J. J. S. Fernandes (Eds.), Atenção à saúde de populações vulneráveis (pp. 1-25). Barueri, SP: Manole.

Basaglia, F. (1979). Psiquiatria Alternativa: Contra o pessimismo da razão, o otimismo da prática. São Paulo, SP: Brasil Debates.

Bergamaschi, F. P. R., Teles, S. A., Souza, A. C. S., \& Nakatami, A. Y. K. (2012). Reflexões acerca da integralidade nas reformas sanitária e agrária. Texto \& Contexto Enfermagem, 21(3), 667674. doi:10.1590/S0104-07072012000300023

Bergamasco, S. M. P., \& Norder, L. A. (1996). O que são assentamentos rurais. São Paulo, SP: Brasiliense.

Bosi, M. L. M., Melo, A. K. D. S., Carvalho, L. B., Ximenes, V. M., \& Godoy, M. G. C. (2014). Determinantes sociais em saúde (mental): Analisando uma experiência não governamental sob a ótica de atores implicados. Revista Brasileira de Epidemiologia, 17(Supl. 2), 126-135. doi:10.1590/1809-4503201400060011

Breilh, J. (2010). Las tres 'S'de la determinación de la vida: 10 tesis hacia una visión crítica de la determinación social de la vida y la salud. In $\mathrm{N}$. Nogueira (Ed.), Determinação social da saúde e reforma sanitária (pp. 87-125). Rio de Janeiro, RJ: Centro de Estudos Brasileiros de Saúde.

Budó, M. D. L. D., \& Saupe, R. (2005). Modos de cuidar em comunidades rurais: A cultura permeando o cuidado de enfermagem. Texto Contexto Enfermagem, 14(2), 177-185. doi:10.1590/ S0104-07072005000200004

Buss, P. M., \& Pellegrini, A., Filho. (2007). A saúde e seus determinantes sociais. Physis, 17(1), 77-93. doi:10.1590/S0103-73312007000100006

Cartier, R., Barcellos, C., Hübner, C., \& Porto, M. F. (2009). Vulnerabilidade social e risco ambiental: Uma abordagem metodológica para avaliação de injustiça ambiental. Cadernos de Saúde Pública, 25(12), 2695-2704.

Confederação Nacional dos Trabalhadores da Agricultura. (2013). Relatório da "Escuta Itinerante: Acesso dos povos do Campo e da Floresta ao SUS". Recuperado em http://www.contag.org. br/index.php? modulo $=$ portal $\&$ acao $=$ interna\&co dpag $=101 \& i d=9668 \&$ data $=11 / 06 / 2014 \& n w=1$ $\& m t=1 \& i n=1$

Costa, M. G. S. G., Dimenstein, M., \& Leite, J. F. (2014). Condições de vida, gênero e saúde mental entre trabalhadoras rurais assentadas. 
Estudos de Psicologia (Natal), 19(2), 145-154. doi:10.1590/S1413-294X2014000200007

Dimenstein, M. (2013). La reforma psiquiátrica y el modelo de atención psicosocial en Brasil: en busca de cuidados continuados e integrados en salud mental. Revista CS, (11), 43-72.

Dimenstein, M., Leite, J., Macedo, J. P., Borges, J. A., Sabadini, M. A., Gomes, M. G., ...Belarmino, V. (2014). Condições de vida e saúde mental em assentamentos de reforma agrária no nordeste brasileiro. In P. Santana \& P. Nossa, P. (Eds.), A Geografia da Saúde no cruzamento de saberes (pp. 113-116). Coimbra, Portugal: Grupo de Investigação em Geografia da Saúde.

Dimenstein, M., Lima, A. I., \& Macedo, J. P. (2013). Integralidade em saúde mental: Coordenação e continuidade de cuidados na Atenção Primária. In S. Paulon \& R. Neves. (Eds.), Saúde Mental na Atenção Básica: A territorialização do cuidado. Porto Alegre: RS: Sulina.

Escola de Saúde Pública de Minas Gerais. (2014). Cuidados em Saúde Mental: Diálogos entre o MST e o SUS. Belo Horizonte, MG: Autor.

Figueiredo, M. D., \& Campos, R. O. (2009). Saúde Mental na atenção básica à saúde de Campinas, SP: Uma rede ou um emaranhado? Ciência \& Saúde Coletiva, 14(1), 129-138. doi:10.1590/ S1413-81232009000100018

Fleury-Teixeira, P., \& Bronzo, C. (2010). Determinação social da saúde e política. In N. Nogueira (Ed.), Determinação social da saúde e reforma sanitária (pp. 37-59). Rio de Janeiro, RJ: Centro de Estudos Brasileiros de Saúde.

Franco, T. B., \& Merhy, E. E. (2013). A produção imaginária da demanda e o processo de trabalho em saúde. In T. B. Franco \& E. E. Merhy, Trabalho, produção do cuidado e subjetividades em saúde (pp. 199-213). São Paulo, SP: Hucitec.

Garbois, J. A., Sodré, F., \& Dalbello-Araujo, M. (2014). Determinantes sociais da saúde: O "social” em questão. Saúde Sociedade, 23(4), 11731182. doi:10.1590/S0104-12902014000400005

Garrafa, V., \& Cordon Portillo, J. A. (2009). Determinantes sociais da doença. Saúde Debate, 33(83), 388-397.

Guimarães, R. B., Pickenhayn, J. A., \& Lima, S. C. (2014). Geografia e saúde sem fronteiras. Uberlândia, MG: Assis.
Helfand, S. M., Rocha, R., \& Vinhais, H. E. (2009). Pobreza e desigualdade de renda no Brasil rural: Uma análise da queda recente. Pesquisa e Planejamento Econômico, 39(1), 59-80.

Juliano, M. C. C., \& Yunes, M. A. M. (2014). Reflexões sobre rede de apoio social como mecanismo de proteção e promoção de resiliência. Ambiente \& Sociedade, 17(3), 135-154.

Kageyama, A., \& Hoffmann, R. (2006). Pobreza no Brasil: Uma perspectiva multidimensional. Economia e Sociedade, 15(1), 26.

Koga, D. (2015). Territórios de vivência em um país continental. Serviço Social \& Saúde, 14(119), 9-26.

Lara, M. O., Brito, M. J. M., \& Rezende, L. C. (2012). Aspectos culturais das práticas dos Agentes Comunitários de Saúde em áreas rurais. Revista da Escola de Enfermagem USP, 46(3), 673-680. doi:10.1590/S0080-62342012000300020

Lima, E. M. F. A. de, \& Yasui, S. (2014). Territórios e sentidos: Espaço, cultura, subjetividade e cuidado na atenção psicossocial. Saúde Debate, 38(102), 593-606.

Merhy, E. E. (2013). Um ensaio sobre o médico e suas valises tecnológicas: Contribuições da análise do território tecnológico do trabalho vivo em ato em saúde para compreender as reestruturações produtivas do setor saúde. In T. B. Franco \& E. E. Merhy, Trabalho, produção do cuidado e subjetividades em saúde (pp. 95-108). São Paulo, SP: Hucitec.

Ministerio de Sanidad y Politica Social. (2011). Rehabilitación psicosocial de pesonas con trastorno mental grave. Pautas de intervención en el domicilio y programas para contextos rurales. Castilla-La Mancha, España: Autor.

Ministério da Saúde. (2011). Política nacional de saúde integral às populações do campo e da floresta. Brasília, DF: Secretaria-Geral da Presidência.

Nogueira, N. (2010). Determinação social da saúde e reforma sanitária. Rio de Janeiro, RJ: Centro de Estudos Brasileiros de Saúde.

Oliveira, E. M. de, Felipe, E. A., Santana, H. da S., Rocha, I. H., Magnabosco, P., \& Figueiredo, M. A de C. (2015). Determinantes sócio-históricos do cuidado na Estratégia Saúde da Família: A perspectiva de usuários da área rural. Saúde $e$ Sociedade, 24(3), 901-913. 
Pinheiro, T. M., Silva, J. M., Carneiro, F., Faria, H., \& Silva, E. (2009). Saúde no campo. In A. Miranda (Coord.), Cadernos de textos da $1^{a}$ Conferência Nacional de Saúde Ambiental. (pp. 25-29). Brasília, DF: Associação Brasileira de Saúde Coletiva.

Rigotto, R. M., Carneiro, F., Ferreira, M. A., Rocha, M., Ferreira, M., Pessoa, V., ...Teixeira, M. M. (2012). O verde da economia no campo: Desafios à pesquisa e às políticas públicas para a promoção da saúde no avanço da modernização agrícola. Ciência \& Saúde Coletiva, 17(6), 1533 1542. doi:10.1590/S1413-81232012000600017

Rocha, S. (2006). Pobreza e indigência no Brasil Algumas evidências empíricas com base na PNAD 2004. Nova Economia, 16(5), 265-299.

Santos, M. (2008). Por uma outra globalização: Do pensamento único à consciência universal. Rio de Janeiro, RJ: Record

Silva, F. C. M., Deus, G. G., Blumm, I. M. L., Souto, K. M. B., Silva, M. G. C. S., Lied, T. B., ...Corrêa, V. S. (2015). A Política Nacional de Saúde Integral das populações do campo, da floresta e das águas e o ambiente. In Ministério da Saúde, Saúde e ambiente para as populações do campo, da floresta e das águas (pp. 9-24). Brasília, DF: Ministério da Saúde.

Silva, V. H F. da, Dimenstein, M., \& Leite, J. F. (2013). O cuidado em saúde mental em zonas rurais. Mental, 10(19), 267-285.

Silva, D. F., \& Santana, P. R. (2012). Transtornos mentais e pobreza no Brasil: Uma revisão sistemática. Tempus - Actas de Saúde Coletiva, 175 185.

Souza, D. O. de, Silva, S. E. V., \& Silva, N. O. de. (2013). Determinantes sociais da saúde: Reflexões a partir das raízes da" questão social". Saúde e Sociedade, 22(1), 44-56.
Tambellini, A. T., \& Schütz, G. E. (2009). Contribuição para o debate do Cebes sobre a "Determinação Social da Saúde": Repensando processos sociais, determinações e determinantes da saúde. Saúde em Debate, 33(83), 371-379.

Uchoa, A. D. C., Souza, E. L., Spinelli, A. F. S., Medeiros, R. G. D., Peixoto, D. C. D. S., Silva, R. A. R. D., ...Rocha, N. S. P. (2011). Avaliação da satisfação do usuário do Programa de Saúde da Família na zona rural de dois pequenos municípios do Rio Grande do Norte. Physis, 21(3), 1061-1076. doi:10.1590/ S0103-73312011000300016

Vasconcelos, E. M. (2013). Manual [de] ajuda e suporte mútuos em saúde mental: Para facilitadores, trabalhadores e profissionais de saúde e saúde mental. Rio de Janeiro, RJ: Escola do Serviço Social da Universidade Federal do Rio de Janeiro.

World Health Organization. (2003). Social determinants of health: The solid facts. Recuperado em http://www.euro.who.int/_ data/assets/pdf_ file/0005/98438/e81384.pdf

World Health Organization. (2010). Mental health and development: Targeting people with mental health conditions as a vulnerable group. Recuperado em http://apps.who.int/iris/bitstream/10665/44257/1/9789241563949_eng.pdf 